\title{
Metabonomic analysis of exhaled breath condensate in adults by nuclear magnetic
} resonance spectroscopy

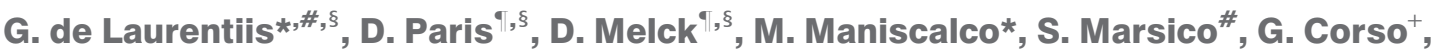 \\ A. Motta ${ }^{\top}$ and M. Sofia*
}

ABSTRACT: Exhaled breath condensate (EBC) is a noninvasive method for the study of airway lining fluid. Nuclear magnetic resonance (NMR) spectroscopy can provide biochemical profiles of metabolites in biological samples. The aim of the present study was to validate the NMR metabonomic analysis of EBC in adults, assessing the role of pre-analytical variables (saliva and disinfectant contamination) and the potential clinical feasibility.

In total, 36 paired EBC and saliva samples, obtained from healthy subjects, laryngectomised patients and chronic obstructive pulmonary disease patients, were analysed by means of ${ }^{1} \mathrm{H}-\mathrm{NMR}$ spectroscopy followed by principal component analysis. The effect on EBC of disinfectant, used for reusable parts of the condenser, was assessed after different washing procedures. To evaluate intra-day repeatability, eight subjects were asked to collect EBC and saliva twice within the same day.

All NMR saliva spectra were significantly different from corresponding EBC samples. EBC taken from condensers washed with recommended procedures invariably showed spectra perturbed by disinfectant. Each EBC sample clustered with corresponding samples of the same group, while presenting intergroup qualitative and quantitative signal differences $(94 \%$ of the total variance within the data).

In conclusion, the nuclear magnetic resonance metabonomic approach could identify the metabolic fingerprint of exhaled breath condensate in different clinical sets of data. Moreover, metabonomics of exhaled breath condensate in adults can discriminate potential perturbations induced by pre-analytical variables.

KEYWORDS: Chronic obstructive pulmonary disease, exhaled breath condensate, metabonomics, nuclear magnetic resonance, principal component analysis, saliva

\begin{abstract}
E xhaled breath condensate (EBC) is a simple, noninvasive and useful tool to study the biochemical and inflammatory molecules in the airway lining fluid [1]. Obtained by cooling exhaled air from spontaneous breathing, EBC predominantly contains water vapour and collects volatile and nonvolatile substances from the lower airways [2]. As such, it can also be considered a matrix for analysis of environmental toxicants and for evaluation of exposure monitoring [3]. Very few data are available on EBC metabolite composition; often single inflammatory molecules are analysed by ELISA and spectroscopic methods.
\end{abstract}

Nuclear magnetic resonance (NMR) studies molecules by recording the interaction of radiofrequency electromagnetic radiation with the nuclei placed in a strong magnetic field. A single nucleus in a molecule can be "observed" by monitoring the corresponding line in an NMR spectrum, and the various parameters of that line (frequency, splitting, linewidth and amplitude) can be used to determine the molecular structure, conformation and dynamics of biomolecules. In principle, assignment (i.e. identification) of NMR lines could be possible by comparing the observed chemical shifts (i.e. the positions in a spectrum) with reference data, spreading out the spectra peaks in two dimensions. Recently, NMR has been applied to biofluids to probe the metabolic status [4-7], and to investigate different diseases [8-11].

The presence of a discriminating element in a series of spectra (i.e. the spectroscopic space) is often undetectable due to the inherent complexity, and is better highlighted by multivariate analysis (principal component analysis; PCA),
AFFILIATIONS

*Dept of Respiratory Medicine, Azienda Ospedaliera Monaldi, University of Naples "Federico II", "Dept of Respiratory Medicine, Azienda Ospedaliera Monaldi, Second University of Naples,

"Institute of Biomolecular Chemistry, Consiglio Nazionale delle Ricerche, Pozzuoli, Naples,

${ }^{+}$Dept of Biomedical Sciences, University of Foggia, Foggia, Italy.

${ }^{\S}$ These authors contributed equally to this work.

\section{CORRESPONDENCE}

G. de Laurentiis

Dept of Respiratory Medicine Azienda Ospedaliera Monaldi Via L. Bianchi 80131 Naples Italy Fax: 390815453213 E-mail: g.delaurentiis@hotmail.it

Received

May 112008

Accepted after revision:

July 142008

\section{SUPPORT STATEMENT}

G. de Laurentiis was supported by the Second University of Naples via a PhD fellowship entitled "Applied cardiorespiratory pathophysiology and biotechnology". D. Paris received a fellowship from the Telethon Institute of Genetics and Medicine (Naples, Italy).

STATEMENT OF INTEREST None declared. 
which carefully identifies hidden phenomena and trends in ensembles of spectra [12]. The application of PCA to a group of spectra can immediately show whether all spectra behave similarly or fall apart into different groups.

Recently, EBC of asthmatic children has been investigated by NMR and statistical analysis [13]. To date, there are several recommendations on the methodological approach to EBC collection, but its standardisation is not completely defined, as most inflammatory mediators, obtained through tracheostomies, are similar to those collected in the mouth [14, 15].

The aims of the present study were: 1) to validate the NMR metabonomic approach to analysis of EBC in adults, assessing the role of pre-analytical variables (saliva and disinfectant contamination) potentially influencing EBC and evaluating the stability and reproducibility of samples; and 2) to evaluate the possibility of discriminating healthy subjects (HS) from patients with airway disease.

\section{MATERIALS AND METHODS \\ Subjects}

A total of 36 paired EBC and saliva samples were collected from the following groups of subjects: 12 HS (nine males, mean age $55.6 \pm 7.2 \mathrm{yrs}$ ); 12 laryngectomised patients (nine males, mean age $60.2 \pm 6.2 \mathrm{yrs}$ ); and 12 patients affected by chronic obstructive pulmonary disease (COPD; 11 males, mean age 64.9 $\pm 5.7 \mathrm{yrs}$ ).

All HS were nonsmokers, while the laryngectomised patients (who provided samples through a stoma, bypassing the pharynx entirely) and the COPD patients were ex-smokers (at least 24 months since smoking). All subjects presented no occupational or other pronounced exposure to organic solvents. The laryngectomised patients had been previously treated by laryngectomy for laryngeal carcinoma $>1$ yr prior (range 1218 months) and did not have a history of chronic respiratory disease or recurrent exacerbations. COPD patients had received diagnosis in the past according to the Global Initiative for Chronic Obstructive Lung Disease guidelines [16]. The COPD anthropometric characteristics are summarised in table 1 . None of the patients were on regular systemic or inhaled corticosteroid treatment. They were asked not to use long-acting $\beta_{2}$-agonist and anticholinergic agents for $\geqslant 12$ and $24 \mathrm{~h}$, respectively, before EBC collection.

All subjects were free from upper and/or lower airway infection for $\geqslant 4$ weeks before the EBC collection. They refrained from food intake for $\geqslant 4 \mathrm{~h}$ before the test and from alcoholic drinks for $\geqslant 18 \mathrm{~h}$ before EBC collection. In laryngectomised patients, lower respiratory tract secretions were actively managed by selfsuctioning and cleaning before each EBC collection.

To assess within-day repeatability, eight subjects (four HS and four COPD patients) were asked to collect EBC and saliva twice within the same day (at times $0 \mathrm{~h}$ and $12 \mathrm{~h}$ ).

All subjects gave informed consent and the study protocol was approved by the Ethics Committee of the Monaldi Hospital (Naples, Italy).

\section{EBC sampling}

EBC was collected using an EcoScreen condenser (Jaeger, Würzburg, Germany) as previously described [13]. Briefly, all subjects breathed through a mouthpiece (laryngectomised patients provided samples through the stoma) and a twoway nonrebreathing valve, which also served as a saliva trap, at normal frequency and tidal volume, while sitting comfortably and wearing a nose-clip for a period of $15 \mathrm{~min}$. They maintained a dry mouth during collection by periodically swallowing excess saliva.

Condensate samples (3-4 mL) were immediately transferred into glass vials of $10 \mathrm{~mL}$ volume, closed with $20-\mathrm{mm}$ butyl rubber lined with polytetrafluoroethylene septa, and crimped with perforated aluminium seals. Volatile substances, possibly deriving from extrapulmonary sources [17, 18, 19], were removed by a gentle stream of nitrogen before sealing. Nitrogen was applied for a variable time (1, 3, 5, 10, 15 and $20 \mathrm{~min})$; no difference was observed with spectra acquired after $1 \mathrm{~min}$ nitrogen exposure, but since such an interval appeared to be too short to avoid systematic errors, a 3-min interval was chosen. Nitrogen was used because the concentration of volatile

\section{TABLE 1 Anthropometric characteristics of 12 patients affected by chronic obstructive pulmonary disease}

\begin{tabular}{|c|c|c|c|c|c|c|c|c|}
\hline Patient & Age yrs & Sex & $\mathrm{BMI} \mathbf{k g} \cdot \mathrm{m}^{-2}$ & FEV $1 \%$ pred & FEV $1 \mathrm{~L} \cdot \mathrm{s}^{-1}$ & $\mathrm{FVC} \mathrm{L} \cdot \mathrm{s}^{-1}$ & FEV $_{1} /$ FVC $\%$ & GOLD stage \\
\hline 1 & 64 & $M$ & 26.6 & 40 & 0.95 & 2.35 & 40.3 & III \\
\hline 3 & 66 & M & 27.1 & 50 & 1.35 & 2.98 & 45.1 & III \\
\hline 4 & 69 & M & 29.7 & 61 & 1.59 & 3.15 & 50.3 & $\|$ \\
\hline 5 & 75 & $M$ & 33.5 & 30 & 0.90 & 2.15 & 41.9 & IV \\
\hline 8 & 66 & $M$ & 25.9 & 70 & 1.74 & 2.89 & 60.1 & 1 \\
\hline 9 & 71 & $M$ & 26.0 & 38 & 0.90 & 1.89 & 47.4 & III \\
\hline 10 & 70 & $\mathrm{~F}$ & 30.1 & 34 & 0.90 & 2.16 & 41.7 & III \\
\hline 11 & 56 & $M$ & 24.5 & 30 & 0.89 & 2.35 & 38.0 & IV \\
\hline 12 & 58 & $M$ & 25.3 & 60 & 1.75 & 3.21 & 54.6 & $\|$ \\
\hline
\end{tabular}

BMI: body mass index; FEV1: forced expiratory volume in one second; \% pred: \% predicted; FVC: forced vital capacity; GOLD: Global Initiative for Chronic Obstructive Lung Disease; M: male; F: female. FEV1, FVC and FEV1/FVC were measured after bronchodilatation inhalation test. 
solutes in EBC is dependent on their distribution between the saliva, exhaled air and droplets, and the condensate. This distribution can be altered by multiple factors, including minute ventilation, salivary $\mathrm{pH}$, solubility, temperature and sample preparation [20]. Therefore, spectral differences may depend upon uncontrollable variables that prevent reliable quantification. The nitrogen stream also removes oxygen from solutions. Such a procedure, used for NMR protein structure determination [21], together with freezing of sealed samples in liquid nitrogen, immediately "quenches" metabolism at the collection time, and prevents any metabolic decay [8]. Samples were then stored at $80^{\circ} \mathrm{C}$ until NMR analysis. Drying of the samples was avoided to circumvent irreversible solute precipitation and/or formation of insoluble aggregates, which were observed upon dissolving the dried condensate for NMR measurements.

\section{Pre-analytical preparation of EBC condenser reusable parts} Before and after collection of each EBC sample, the reusable parts of the condenser (valve, salivary trap and lamellar condenser) were disinfected for 15 min using a solution of a $1.5 \%$ freshly prepared chemical agent (Descogen ${ }^{\mathrm{TM}}$; FILT $\mathrm{GmbH}$, Berlin, Germany), and repeatedly flushed with water following the manufacturer's guidelines. To completely eliminate the disinfectant, parts already disinfected and washed were thoroughly rinsed for $15 \mathrm{~min}$ with pure grade ethanol $(96 \%)$, thereafter exhaustively soaked with deionised distilled water for $15 \mathrm{~min}$ and dried under vacuum at $50^{\circ} \mathrm{C}$.

\section{Salivary collection}

On the same day as EBC collection, a salivary sample was taken. To avoid any interference from exogenous agents into the oral environment, the patients were asked to collect all saliva available $(\sim 2-4 \mathrm{~mL})$, i.e. "whole" saliva expectorated from the mouth, into a plastic universal tube immediately after waking in the morning. As previously described by SILWOOD et al. [10], each patient was requested to refrain completely from oral activities (i.e. eating, drinking, tooth brushing, oral rinsing, smoking, etc.) during the short period between awakening and sample collection $(<5 \mathrm{~min})$. Each collection tube contained $15 \mu \mathrm{mol}$ sodium fluoride, sufficient to ensure that metabolites were not generated or consumed via the actions of bacteria or bacterial enzymes present in whole saliva during periods of sample preparation and/or storage [11]. Specimens were transported to the laboratory on ice and immediately centrifuged (at $20,000 \times g$ at $4{ }^{\circ} \mathrm{C}$ for $15 \mathrm{~min}$ ) on their arrival to remove cells and debris. Following this, a gentle nitrogen gas flow was applied for $\sim 5$ min to supernatants, which were then stored at $-80^{\circ} \mathrm{C}$ until measurements were made [11].

The ${ }^{1} \mathrm{H}-\mathrm{NMR}$ profiles of salivary supernatant specimens subjected to analysis immediately after collection into the fluoride-containing tubes and rapid centrifugation were compared with those of the same samples stored as described previously, and no differences were discernible, i.e. none of the criteria investigated changed significantly during these periods of storage.

\section{Sample preparation for NMR analysis}

EBC samples were rapidly defrosted. To provide a field frequency lock, $70 \mu \mathrm{L}$ of a deuterium oxide $\left(\mathrm{D}_{2} \mathrm{O}\right)$ solution, containing $1 \mathrm{mM}$ sodium 3-trimethylsilyl $\left(2,2,3,3-{ }^{2} \mathrm{H}_{4}\right)$ propionate
(TSP) as a chemical shift reference for ${ }^{1} \mathrm{H}$ spectra and sodium azide at $3 \mathrm{mM}$, was added to $630 \mu \mathrm{L}$ of condensate, thus making $700 \mu \mathrm{L}$ total volume. Saliva samples were rapidly defrosted and $70 \mu \mathrm{L}$ of reference standard solution $\left(\mathrm{D}_{2} \mathrm{O}-\mathrm{TSP}\right)$ was added to $630 \mu \mathrm{L}$ of sample.

\section{NMR measurements}

One-dimensional (1D) spectra were recorded on a Bruker Avance spectrometer (Bruker BioSpin $\mathrm{GmbH}$, Rheinstetten, Germany) operating at a frequency of $600.13 \mathrm{MHz}\left({ }^{1} \mathrm{H}\right)$ and equipped with a TCI CryoProbe ${ }^{\mathrm{TM}}$ (Bruker BioSpin $\left.\mathrm{GmbH}\right)$, at a probe temperature of $27^{\circ} \mathrm{C}$. The water resonance was suppressed by using the noesypresat pulse sequence, called noesypr1d according to the manufacturers. It has the form $\mathrm{RD}-90^{\circ}-\mathrm{t}-90^{\circ}-\mathrm{t}_{\mathrm{m}}-\mathrm{ACQ}$, where $\mathrm{RD}$ is a relaxation delay, $\mathrm{t}$ a short delay, $90^{\circ}$ represents a $90^{\circ}$ radio frequency pulse, $t_{m}$ the mixing time and ACQ the data acquisition period. In the present study acquisition conditions, the carrier frequency $(\mathrm{O} 1)$ value was set on the water resonance, the saturation power was $62 \mathrm{~dB}$, $\mathrm{t}$ was $4 \mu \mathrm{s}, \mathrm{t}_{\mathrm{m}}$ was $100 \mathrm{~ms}$, the spectral amplitude was $7002.8 \mathrm{~Hz}$, the time domain was $16 \mathrm{~K}, \mathrm{RD}$ was $2.0 \mathrm{~s}$ and the number of transients was 256 . This resulted in a total acquisition time of 14 min per sample. For processing, a line broadening of $0.6 \mathrm{~Hz}$ was applied and a real spectrum size of $32 \mathrm{~K}$ was used. Spectra were referred to TSP, assumed to resonate at a $\delta$ of $0.00 \mathrm{ppm}$.

\section{Statistical analysis}

High-resolution ${ }^{1} \mathrm{H}-\mathrm{NMR}$ spectra were automatically datareduced to 200 integral segments ("buckets"), each of 0.02 ppm, using the AMIX software package (Bruker BioSpin GmbH). The resulting integrated regions were imported into the SIMCA package (Umetrics, Umea, Sweden) and used for statistical analysis and pattern recognition. Before pattern recognition analysis, each integral region is usually normalised to the sum of all integral regions of each spectrum; however, because of the presence of contaminant peaks, each bucket was normalised to the TSP peak of known concentration for a reference region of between 0.014 and $-0.014 \mathrm{ppm}$. The correctness of the approach was tested by comparing the results with those obtained by referring to the sum of all integral regions of each contaminantfree spectrum. No significant difference was observed between the two approaches; therefore, pattern recognition analysis was reliable with normalisation to TSP. Data were preprocessed with the Centering scaling and then processed with PCA and partial least squares discriminant analysis (PLS-DA).

\section{RESULTS}

\section{Spectral differences between EBC and saliva}

Figure 1 represents spectra of saliva (fig. $1 \mathrm{a}, \mathrm{c}$ and e) and EBC samples (fig. 1b, d and f) from one HS (fig. 1a and b), one laryngectomised patient (fig. 1c and d) and one COPD patient (fig. 1e and f). Saliva spectra were highly different from corresponding EBC samples and were notably dissimilar between patients: a visual examination establishes a correspondence between spectra from a HS (fig. 1a) and a laryngectomised patient (fig. 1c), but a difference from the COPD spectrum (fig. 1e), which shows sharper lines. The most intense signals in the $0.0-3.2 \mathrm{ppm}$ region of saliva were assigned according to previous studies [11, 22]. Resonance assignment was as follows: leucine $\delta \mathrm{CH}_{3} \mathrm{~s}$ (triplet) at $0.96 \mathrm{ppm}$; propionate $\mathrm{\beta CH}_{3}$ at $1.04 \mathrm{ppm}$ (triplet) and $\alpha \mathrm{CH}_{2}$ at $2.19 \mathrm{ppm}$ 
a)

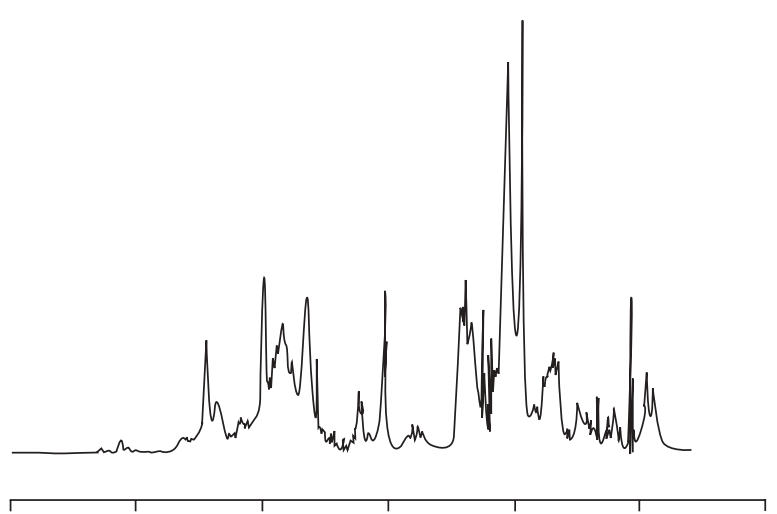

c)

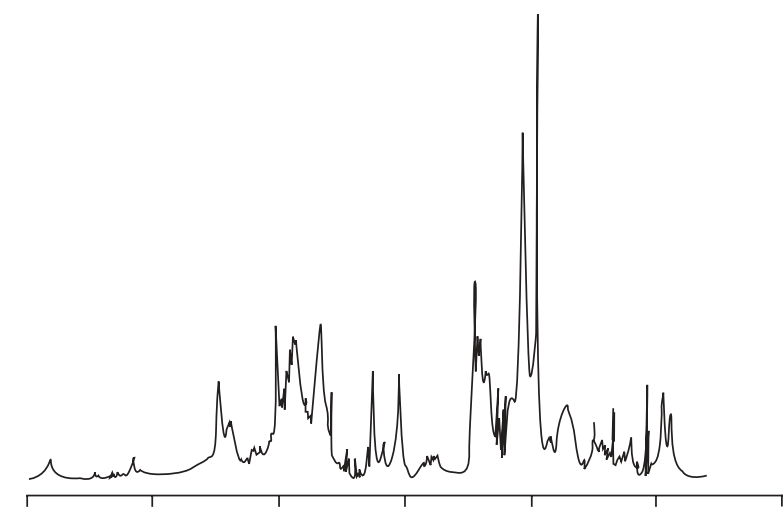

e)

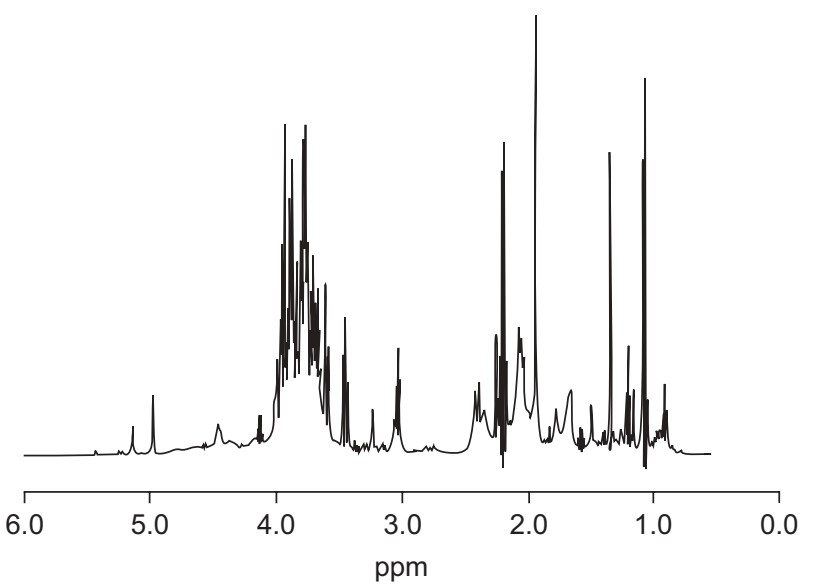

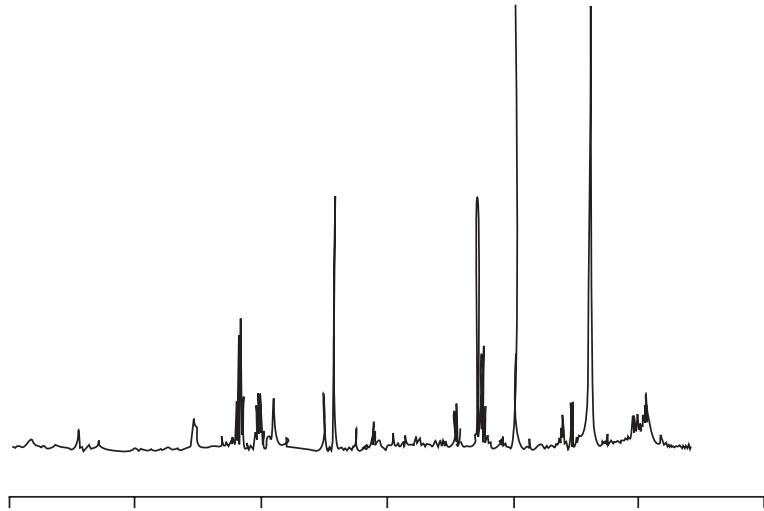

d)

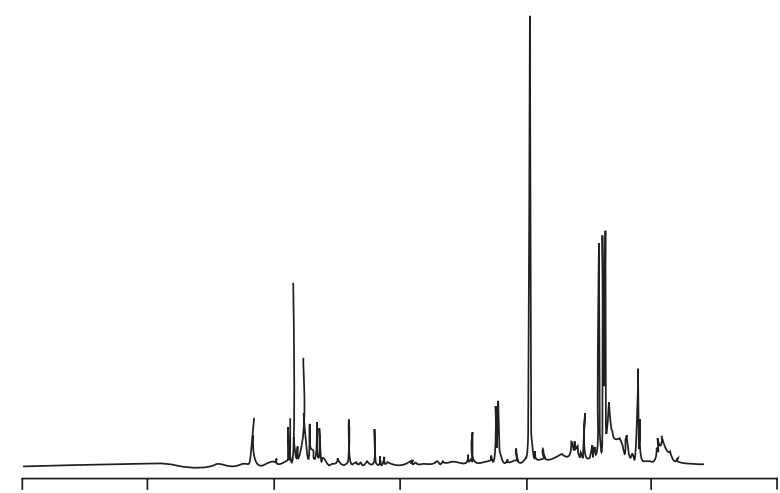

f)

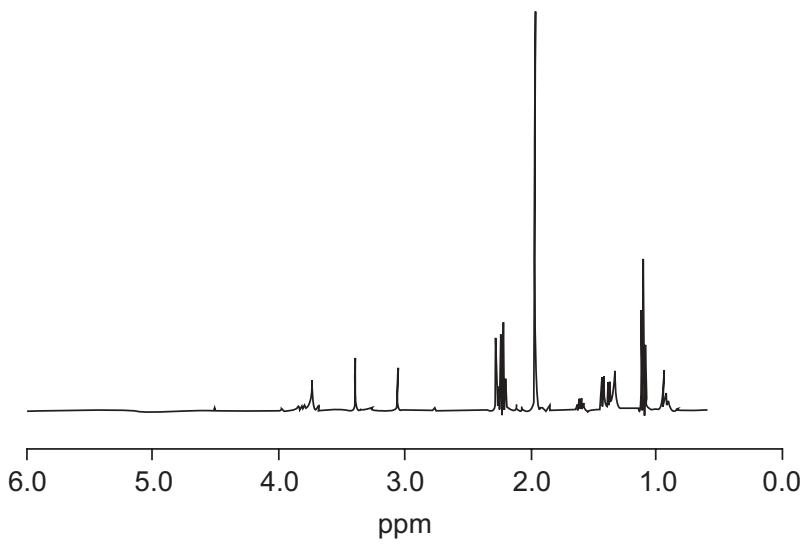

FIGURE 1. Representative one-dimensional ${ }^{1} \mathrm{H}$-nuclear magnetic resonance spectra of saliva ( $\mathrm{a}, \mathrm{C}$ and e) and exhaled breath condensate (EBC; $b, d$ and $\left.\mathrm{f}\right)$ samples from healthy ( $a$ and b), laryngectomised ( $c$ and d) and chronic obstructive pulmonary disease (e and f) patients. The group of signals centred at 3.8 ppm in saliva spectra originates from carbohydrates and is not visible in the corresponding EBC spectra.

(quartet); lactate $\mathrm{CCH}_{3}$ at $1.32 \mathrm{ppm}$ (doublet) and $\alpha \mathrm{CH}$ at $4.11 \mathrm{ppm}$ (quartet); threonine $\mathrm{\gamma CH}_{3}$ at $1.36 \mathrm{ppm}$ (doublet); alanine $\mathrm{\beta CH}_{3}$ at $1.47 \mathrm{ppm}$ (doublet) and $\alpha \mathrm{CH}$ at $4.20 \mathrm{ppm}$ (quartet); acetate $\beta \mathrm{CH}_{3}$ (singlet) at $1.93 \mathrm{ppm} ; \beta \mathrm{CH}_{2}$ of glutamate and glutamine at $2.10 \mathrm{ppm}$ (multiplet); $\beta_{\mathrm{CH}}$ of pyruvate at $2.37 \mathrm{ppm}$ (singlet); $\alpha, \mathrm{BCH}_{2}$ of succinate at 2.41 ppm (singlet); $\varepsilon \mathrm{CH}_{2}$ of lysine at 3.06 (triplet); $\mathrm{N}^{-\mathrm{CH}_{3} \mathrm{~S} \text { of }}$ choline at $3.16 \mathrm{ppm}$ and of phosphorylcholine at $3.23 \mathrm{ppm}$ (both singlets); and $\mathrm{N}-\mathrm{CH}_{3}$ of taurine at $3.23 \mathrm{ppm}$ (triplet). Signals between 3.3 and 6.0 ppm originate from carbohydrates and were virtually absent in the EBC spectra.
Compared with saliva, EBC spectra presented fewer signals and, as observed for saliva, the COPD patient trace (fig. 1f) appeared to be different from the HS (fig. 1b) and laryngectomised patient (fig. 1d) traces.

Spectral differences between saliva and EBC were verified by PLS-DA analysis. Due to the complete absence of the carbohydrate signals in the EBC spectrum, the region 5.0 to 3.5 ppm was cut out from all spectra, partitioning the region between 3.5 and $0.8 \mathrm{ppm}$. Figure 2 shows the score plots of saliva and EBC samples from all subjects. Considering two 


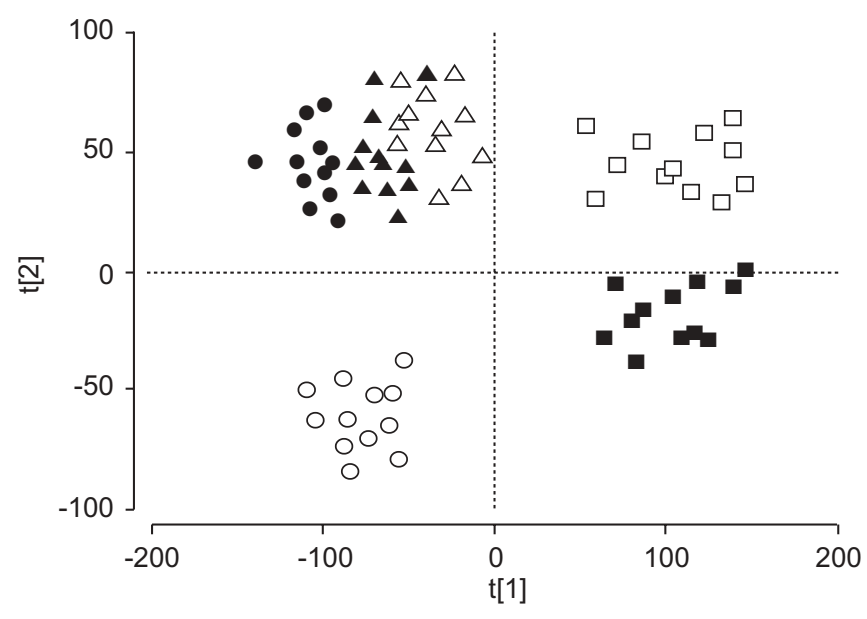

FIGURE 2. Partial least squares discriminant analysis (PLS-DA) scores discrimination for exhaled breath condensate $(\triangle$ : laryngectomised patients; $\Lambda$ healthy subjects (HS); $\bullet$ : chronic obstructive pulmonary disease (COPD) patients) and saliva ( $\square$ : laryngectomised; $\mathbf{\square}$ : HS; O: COPD). All variables were used and two PLS-DA components were retained in the model, obtaining a classification of $\sim 95 \%$. The region 5.0 to $3.5 \mathrm{ppm}$, containing the carbohydrate signals, was cut out from the bucketing, and only the signals between 3.5 and $0.8 \mathrm{ppm}$ were analysed. $\mathrm{t}[1]$ and $\mathrm{t}[2]$ are the first two principal components.

PLS-DA components, it was possible to obtain a sample classification of $\sim 95 \%$ (samples correctly classified into different regions). In particular, while EBC samples were all clustered, the saliva samples of HS, laryngectomised and COPD patients were positioned differently from EBC and from each other. Such a separation comes mostly from signals resonating within the 3.5-2.9 and 2.1-1.7 ppm regions.

a)

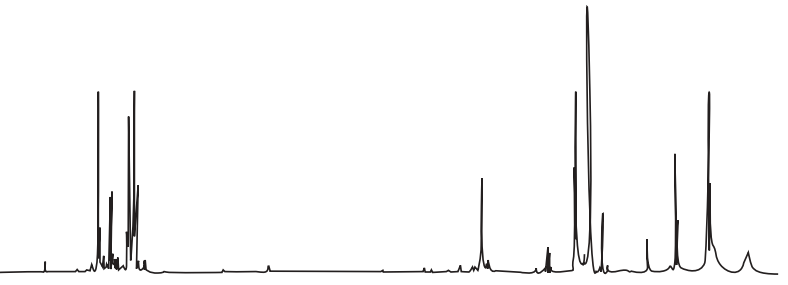

c)

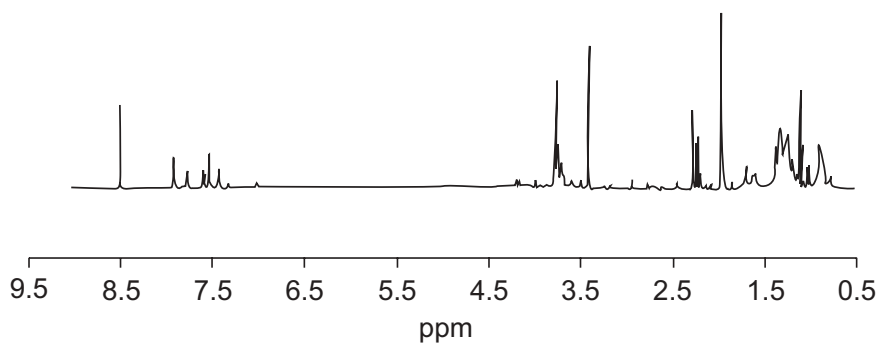

EBC and saliva samples collected from eight subjects twice within the same day (at times $0 \mathrm{~h}$ and $12 \mathrm{~h}$ ) demonstrated good within-day repeatability, showing no evident difference in resonances in the spectra.

\section{Effects of disinfectant contamination on EBC spectra}

Figure 3 shows the 1D ${ }^{1} \mathrm{H}-\mathrm{NMR}$ spectrum of Descogen ${ }^{\mathrm{TM}}$ (fig. 3a) with representative spectra of EBC samples contaminated by the disinfectant because of insufficient washing time (fig. $3 b$ and c). To completely eliminate the disinfectant, parts already disinfected and washed were thoroughly rinsed for 15 min with pure grade ethanol (96\%), thereafter exhaustively soaked with deionised distilled water for $15 \mathrm{~min}$ and dried under vacuum at $50^{\circ} \mathrm{C}$ (fig. $3 \mathrm{~d}$ ).

The resonances of the "saline" components of the disinfectant (citric acid, at $2.66 \mathrm{ppm}$ in the Descogen ${ }^{\mathrm{TM}}$ spectrum (fig. 3a), and pentapotassium bis(peroxymonosulphate) bis(sulphate), highly soluble in water) disappeared completely after partial washing (15 min; fig. 3b). However, minor unknown components, such as those giving signals in the 8.2-7.3 and 1.3$0.7 \mathrm{ppm}$ regions and the signal located at $3.2 \mathrm{ppm}$, appeared to be more persistent even after intense water rinsing $(30 \mathrm{~min}$; fig. 3c). They were completely removed only after the washing procedure using ethanol (fig. 3d).

As the perturbation induced by the disinfectant contamination of EBC samples showed visible signals, two different contaminated sets of 12 EBC samples from all COPD patients were examined after partial washing (15 min, "high Descogen ${ }^{\mathrm{TM}}$ "; fig. 3b); and after intense water rinsing (30 min, "low Descogen "TM"; fig. 3c). Since the region 8.5-7.0 ppm was absent in the "cleaned" EBC spectrum (fig. 3d), as suggested by CARRARO et al. [13], the region 4.5 to $0.5 \mathrm{ppm}$ was used and the lactate signals were excluded

b)

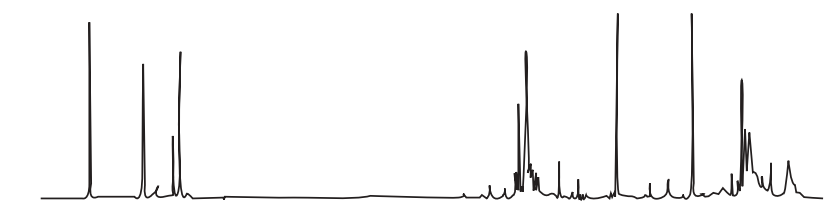

d)

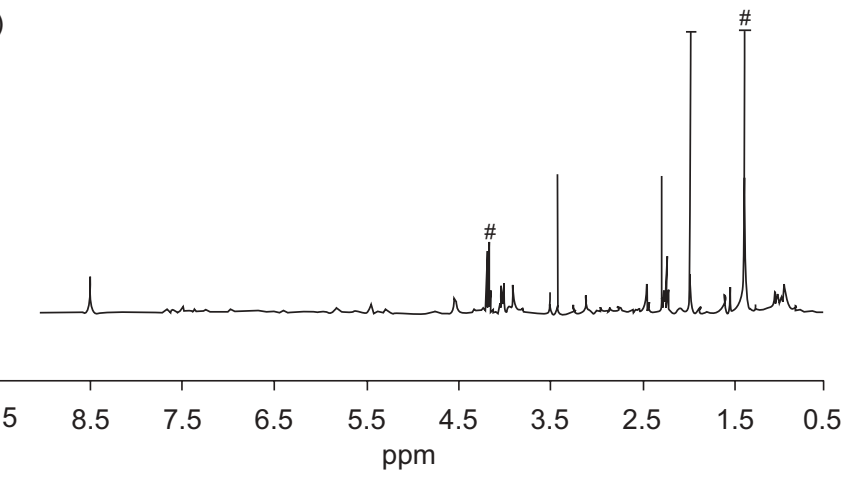

FIGURE 3. Contamination of exhaled breath condensate (EBC) samples by Descogen ${ }^{\mathrm{TM}}$ (FILT GmbH, Berlin, Germany). a) ${ }^{1} \mathrm{H}$-nuclear magnetic resonance spectrum of Descogen ${ }^{\mathrm{TM}}$, compared with b) spectra of EBC samples after partial washing (15 min), and c) intense water rinsing (30 min). d) Contamination was completely removed after the washing procedure using ethanol. The acetate signal at $1.93 \mathrm{ppm}$ was cut in all EBC spectra. a) The vertical scale is one quarter the size of the other spectra. \#: lactate resonances. 

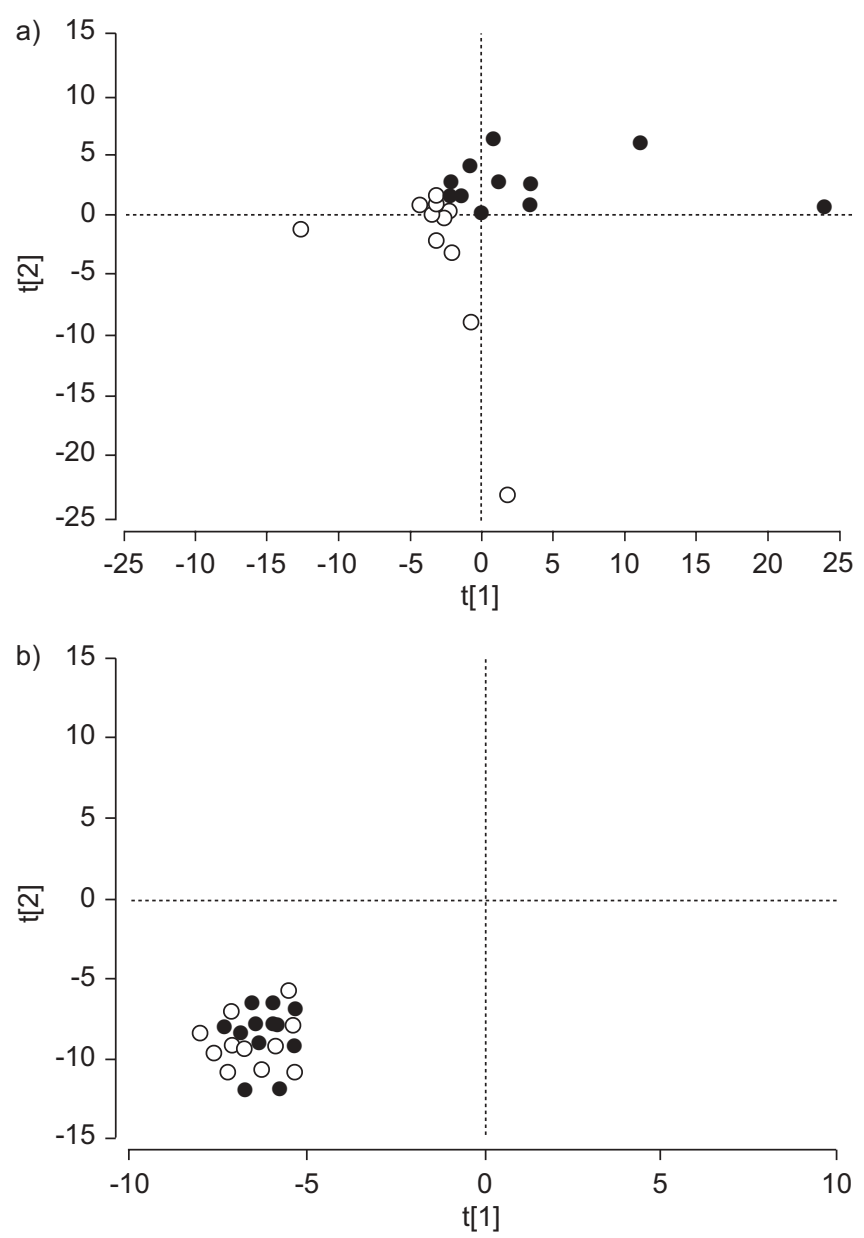

FIGURE 4. Partial least squares discriminant analysis scores discrimination for contaminated exhaled breath condensate (EBC) samples after a) different washing times, and b) selecting the 3.5-2.9 and 2.1-1.7 ppm regions for statistics. b) Exclusion of the most intense Descogen ${ }^{\mathrm{TM}}$ signals (FILT GmbH, Berlin, Germany) allowed a classification of $\sim 94 \%$, as expected from "clean" EBC samples. $\bigcirc$ : high Descogen ${ }^{\mathrm{TM}}$ (15-min rinsing, as in fig. 3b); • low Descogen ${ }^{\mathrm{TM}}$ (30-min rinsing, as in fig. 3c). t[1] and t[2] are the first two principal components.

(fig. 3d). Considering two PLS-DA components, a classification of $\sim 72 \%$ was obtained, with high-Descogen ${ }^{\mathrm{TM}}$ and lowDescogen $^{\mathrm{TM}}$ EBC samples classified in two wide regions (fig. 4a). This suggests that the presence of the disinfectant at variable concentration affects the interpretation and the statistical analysis of the samples. However, if the presence of contaminant is ignored by a careful selection of the spectral regions to be used for statistical analysis, it is possible to correctly classify the samples. In fact, by selecting only the Descogen ${ }^{\mathrm{TM}}$ free regions of the spectra (3.5-2.9 and 2.1-1.7 ppm), all the samples were correctly classified (94\%) in a single region (fig. $4 \mathrm{~b}$ ), as expected from "clean" (i.e. totally Descogen ${ }^{\mathrm{TM}}$-free) EBC samples.

\section{EBC spectral discrimination between HS, laryngectomised and COPD patients}

The 3.5-1.7 ppm region of clean (i.e. Descogen ${ }^{\mathrm{TM}}$-free) $\mathrm{EBC}$ samples was used to investigate the metabolites characterising

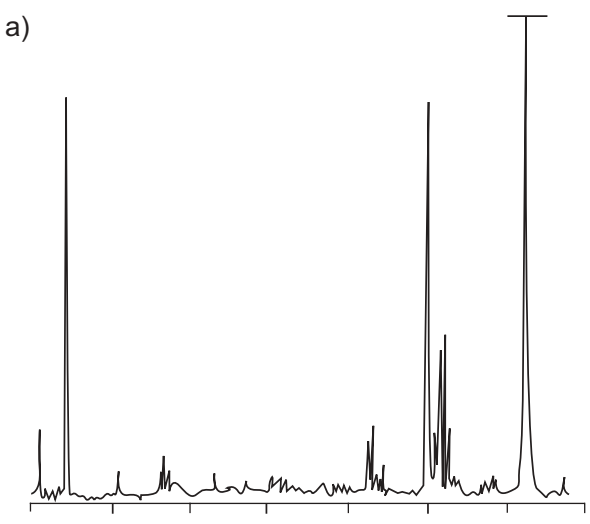

b)

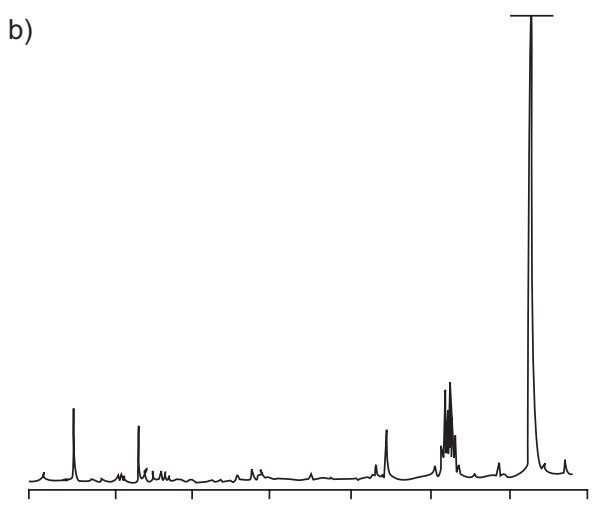

c)

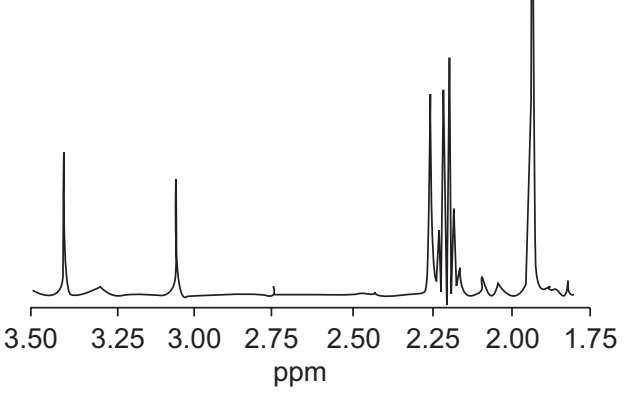

FIGURE 5. Representative ${ }^{1} \mathrm{H}$-nuclear magnetic resonance spectra of contaminant-free exhaled breath condensate samples from a) healthy subjects b) laryngectomised patients and c) chronic obstructive pulmonary disease patients. The acetate singlet at $1.93 \mathrm{ppm}$ is cut by a horizontal bar.

EBC. Figure 5 depicts representative spectra of HS (fig. 5a), laryngectomised patients (fig. 5b) and COPD patients (fig. 5c). Although the region contains few signals, the signals specifically characterise each patient subset, showing both quantitative (signal intensity) and qualitative (signal absence/ presence) differences. Differences in intensity were shown by the signals of: acetate $\mathrm{\beta CH}_{3}$ (singlet) at $1.93 \mathrm{ppm}$; propionate $\alpha \mathrm{CH}_{2}$ at $2.19 \mathrm{ppm}$ (quartet); pyruvate $\beta \mathrm{CH}_{3}$ (singlet) at $2.37 \mathrm{ppm}$; succinate $\alpha, \beta \mathrm{CH}_{2}$ (singlet) at $2.41 \mathrm{ppm}$; glutamine $\gamma \mathrm{CH}_{2}$ (multiplet) at $2.45 \mathrm{ppm}$; choline and phosphorylcholine 


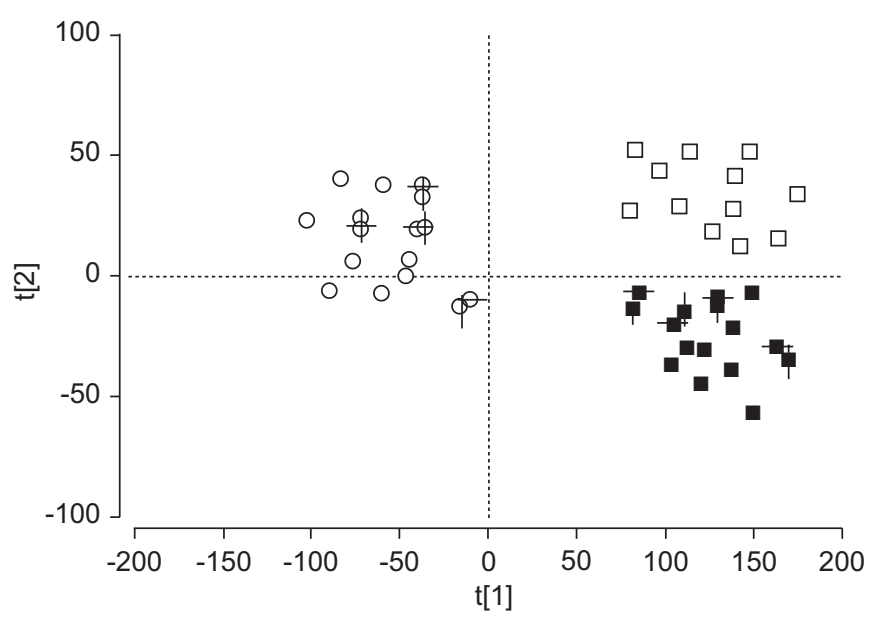

FIGURE 6. Partial least squares discriminant analysis (PLS-DA) scores discrimination for contaminant-free exhaled breath condensate samples. $\mathbf{a}$ : healthy subjects; $\square$ : laryngectomised patients; $\bigcirc$ : chronic obstructive pulmonary disease patients. Two PLS-DA components afforded a clear classification ( $94 \%)$, with all samples correctly classified into three regions. Vertical and horizontal bars refer to samples collected in duplicate. t[1] and t[2] are the first two principal components.

$\mathrm{N}-\mathrm{CH}_{3} \mathrm{~s}$ (singlets) at 3.16 and 3.23 ppm, respectively; methanol $\mathrm{CH}_{3}$ at $3.37 \mathrm{ppm}$ (singlet); and trimethylamine-N-oxide (TMAO) $\mathrm{N}-\mathrm{CH}_{3}$ (singlet) at $3.44 \mathrm{ppm}$, as well as by the singlet at $3.03 \mathrm{ppm}$ that most likely originated from $\mathrm{N}-\mathrm{CH}_{3}$ of creatine/creatinine. Pyruvate was present in the COPD spectrum (fig. 5c) and was very intense in the HS spectrum (fig. 5a), but barely visible in the laryngectomised spectrum (fig. 5b). Succinate was small in the HS spectrum (fig. 5a), bigger in the laryngectomised spectrum (fig. 5b) but absent in the COPD spectrum (fig. 5c). Glutamine was only present in the HS spectrum (fig. 5a). The singlet at 3.03 ppm was only present in the COPD spectrum (fig. 5c). Choline and phosphorylcholine were absent in the COPD spectrum (fig. $5 \mathrm{c}$ ), and TMAO was present in the HS spectrum (fig. 5a), barely seen in the laryngectomised spectrum (fig. 5b) and absent in the COPD spectrum (fig. 5c).

All these differences prompted a clear discrimination of HS, laryngectomised and COPD patients in three separate groups (fig. 6).

\section{DISCUSSION}

The present study demonstrates, for the first time, that NMRbased metabonomics can be used to analyse EBC samples from adults, allowing a clear-cut separation between HS and patients with airway disease.

Although less sensitive than ELISA and mass spectrometry, NMR requires minimal sample preparation with a rapid acquisition time $(\sim 10-15 \mathrm{~min})$ and has a high degree of sensitivity (less than or equivalent to $\mu \mathrm{mol} \cdot \mathrm{L}^{-1}$ levels). Furthermore, it is nondestructive and allows complete detection of observable metabolites ("sample metabolic fingerprint") at a reasonable cost.

The present data show that saliva is significantly different from the EBC samples and that the presence of identical metabolites in EBC and saliva does not hamper discrimination. By selecting the 3.5-0.8 ppm region (thereby excluding the carbohydrate signals absent in EBC), saliva spectra clearly differ from EBC (fig. 2), notwithstanding the presence of some common metabolites (leucine, lactate, propionate, acetate, etc.).

EBC standardising guidelines [2] indicate that it is reasonable to assume that there is some degree of oral contamination of EBC, as saliva contains many of the mediators that are also present in the lower airways. Contamination of EBC is often proved by measuring the amylase level, but such a test is not specific and a negative signal does not completely exclude minute contribution from the mouth. To date, there are no data comparing the metabolic saliva composition and a lower airway derivate such as EBC, mainly because condensate samples have been screened for single, specific biomarkers and not as a whole. Indeed, combined saliva and EBC analysis by a metabonomics method has been recently advocated [19]. In light of these assumptions, the current authors also examined EBC from laryngectomised patients, which may represent a true saliva-free material from the lower airways, showing that in those subjects all saliva spectra strictly differed from corresponding EBC samples. Importantly, all EBC and saliva collected twice within the same day (12 h apart) showed good within-day repeatability (fig. 6). Taken together, the data suggest that saliva contamination may play a minor role in the interpretation of EBC by NMR-based metabonomics.

The influence of external contaminants was also considered, as the International Consensus on EBC recommends special care in the disinfection of reusable parts of condensers [1]. Upon standard cleaning, all EBC spectra presented signals corresponding to unknown inactive substances of the disinfectant. They persisted even after strong and repeated water soaking, and the presence of variable disinfectant concentration upon different cleaning levels may render classification less effective. Complete removal of the disinfectant signals was observed after washing the reusable parts with $96 \%$ ethanol and then rinsing thoroughly with distilled water for $15 \mathrm{~min}$. EBC samples were "spiked" by partially washing the apparatus with water, after treatment with freshly prepared Descogen ${ }^{\mathrm{TM}}$, obtaining different degrees of EBC contamination. Since the citric acid signals were absent after partial washing (fig. 3b), it is important to underline that the potentially toxic saline components of the disinfectant are easily removed from the condenser apparatus by water washing. However, the removal of interfering residual external contaminants is crucial for a correct EBC analysis. There are no data on the influence of residual disinfectant agents of reusable parts of EBC condensers. The influence of residual Descogen ${ }^{\mathrm{TM}}$ on reported biomarker levels was not evaluated by an ELISA method, but the present authors suggest that the potential role of external contamination on the variability of some biomarkers $[23,24]$ should be evaluated.

Significantly, by selecting specific regions of EBC spectra for statistical analysis, an efficient discrimination of samples was obtained. Although separation between HS and COPD patients can be achieved by either forced expiratory volume in one second measurements or clinically, the current authors evaluated the capability of NMR-based metabonomics to separate EBC subjects with airway diseases (COPD) from subjects without respiratory diseases. Five NMR signals appear to 
differentiate "respiratory" (COPD) from "nonrespiratory" (HS and laryngectomised) subjects. As a comparison, CARRARO et al. [13] reported the single acetate signal variation as distinctive in asthmatic children with respect to controls. They hypothesised that acetate increase might be related to increased acetylation of pro-inflammatory proteins in the extracellular space in the airway environment. Furthermore, they found that peaks in 3.23.4 ppm regions of the NMR spectrum of asthmatic children were probably related to oxidised compounds. HEILI-FRADES et al. [25] have reported preliminary data on significant variations between NMR EBC spectra of normal and pathological cases with implications for correlative studies using spectral and clinical classification.

In the present study, by comparing EBC from respiratory (COPD) patients and nonrespiratory (HS and laryngectomised) subjects, as well as acetate, four additional signal variations were found, which are likely to have included the methoxy compounds. It can be speculated that such variations could derive from the increased oxidative stress that is a hallmark of COPD, and these variations are usually investigated in EBC by measuring a limited number of markers [23, 24]. Also, the comparison between HS, laryngectomised and COPD EBC samples showed a clear-cut difference (fig. 5) in the COPD patients compared with the other subjects. Figure 6 depicts a significant statistical difference along $\mathrm{t}[1]$ of COPD patients compared with HS and laryngectomised patients, who are less separated along $\mathrm{t}[2]$. This could be interpreted by the fact that laryngectomised patients were not labelled as COPD before or after surgery; furthermore, mild airflow limitation was detected in only a few subjects (data not shown).

The current authors are aware that the present study relies on a limited number of subjects without a prospective test sample of different groups of subjects, followed by an external validation in a second set of subjects. However, they are collecting more NMR data on EBC in order to build a database to be used, together with other techniques, for prognostic and/ or diagnostic purposes. Moreover, the observed metabolites were not identified in the present study, and the current authors are currently characterising all resonances by using homo- and heteronuclear two-dimensional spectra, and the results will be reported in due course.

In conclusion, nuclear magnetic resonance-based metabonomics can safely be applied to exhaled breath condensate in adults, allowing an unambiguous definition irrespective of natural and/or artificial contaminants. In particular, the current authors report that nuclear magnetic resonance spectra of exhaled breath condensate, collected with a device using a salivary trap, do not show the presence of saliva signals. Furthermore, for the disinfectant medium currently used, a careful selection of the nuclear magnetic resonance region allows a clear statistical classification of samples, even for contaminated exhaled breath condensate samples. Finally, the present results suggest that condensate can be efficiently studied as a whole, and that nuclear magnetic resonance may become a leading diagnostic technique in this field.

\section{ACKNOWLEDGEMENTS}

The authors would like to thank E.P. Castelluccio (IBC-CNR, Pozzuoli, Italy) for computer assistance and M. Mormile
(University of Naples "Federico II", Naples, Italy) for technical support with stomospirometry in laryngectomised patients.

\section{REFERENCES}

1 Kharitonov SA, Barnes PJ. Exhaled markers of inflammation. Curr Opin Allergy Clin Immunol 2001; 1: 217-224.

2 Horváth I, Hunt J, Barnes PJ, et al. Exhaled breath condensate: methodological recommendations and unresolved questions. Eur Respir J 2005; 26: 523-548.

3 Maniscalco M, de Laurentiis G, Pentella C, et al. Exhaled breath condensate as matrix for toluene detection: a preliminary study. Biomarkers 2006; 11: 233-240.

4 Lindon JC, Holmes E, Nicholson JK. Metabonomics in pharmaceutical R\&D. FEBS J 2007; 274: 1140-1151.

5 Nicholson JK, Lindon JC, Holmes E. Metabonomics: understanding the metabolic responses of living systems to pathophysiological stimuli via multivariate statistical analysis of biological NMR spectroscopic data. Xenobiotica 1999; 29: 1181-1189.

6 Nicholson JK, Connelly J, Lindon JC, Holmes E. Metabonomics: a platform for studying drug toxicity and gene function. Nat Rev Drug Discov 2002; 1: 153-161.

7 Beckonert O, Keun HC, Ebbels TMD, et al. Metabolic profiling, metabolomic and metabonomic procedures for NMR spectroscopy of urine, plasma, serum and tissue extracts. Nat Protoc 2007; 2: 2692-2703.

8 Bell JD, Brown JC, Sadler PJ. NMR studies of body fluids. NMR Biomed 1989; 2: 246-256.

9 Holmes E, Foxall PJ, Nicholson JK. Proton NMR analysis of plasma from renal failure patients: evaluation of sample preparation and spectral-editing methods. J Pharm Biomed Anal 1990; 8: 955-958.

10 Silwood CJ, Lynch E, Claxson AW, Grootveld MC. 1H and 13C NMR spectroscopic analysis of human saliva. J Dent Res 2002; 81: 422-427.

11 Grootveld M, Silwood CJ. 1H NMR analysis as a diagnostic probe for human saliva. Biochem Biophys Res Commun 2005; 329: 1-5.

12 Lindon JC, Holmes E, Nicholson JK. Pattern recognition methods and applications in biomedical magnetic resonance. Prog Nucl Magn Reson Spectrosc 2001; 39: 1-40.

13 Carraro S, Rezzi S, Reniero F, et al. Metabolomics applied to exhaled breath condensate in childhood asthma. Am J Respir Crit Care Med 2007; 175: 986-990.

14 Latzin P, Beck J, Bartenstein A, Griese M. Comparison of exhaled breath condensate from nasal and oral collection. Eur J Med Res 2003; 8: 505-510.

15 Chladkova J, Krcmova I, Chladek J, Cap P, Micuda S, Hanzalkova Y. Validation of nitrite and nitrate measurements in exhaled breath condensate. Respiration 2006; 73: 173-179.

16 Rabe KF, Hurd S, Anzueto A, et al. Global strategy for the diagnosis, management, and prevention of chronic obstructive pulmonary disease: GOLD executive summary. Am J Respir Crit Care Med 2007; 176: 532-555.

17 Effros RM. Exhaled breath condensate pH. Am J Respir Crit Care Med 2006; 173: 1047-1048.

18 Effros RM, Hoagland KW, Bosbous M, et al. Dilution of respiratory solutes in exhaled condensates. Am J Respir Crit Care Med 2002; 165: 663-669. 
19 Whittle CL, Fakharzadeh S, Eades J, Preti G. Human breath odors and their use in diagnosis. Ann N Y Acad Sci 2007; 1098: 252-266.

20 Effros RM. Metabolomics in exhaled breath condensates. Am J Respir Crit Care Med 2008; 177: 236.

21 Wüthrich K. NMR of Protein and Nucleic Acids. New York, Wiley and Sons, 1986.

22 Fan TW-M. Metabolite profiling by one- and two-dimensional NMR analysis of complex mixtures. Prog NMR Spectrosc 1996; 28: 161-219.
23 Montuschi P. Exhaled breath condensate analysis in patients with COPD. Clin Chim Acta 2005; 356: 22-34.

24 Montuschi P, Kharitonov SA, Ciabattoni G, Barnes PJ. Exhaled leukotrienes and prostaglandins in COPD. Thorax 2003; 58: 585-588.

25 Heili-Frades S, Peces-Barba G, Rodriguez-Nieto MJ, et al. Metabonomic approach for non invasive diagnosis of inflammatory lung diseases through nuclear magnetic resonance analysis of exhaled breath condensate. Eur Respir J 2007; 30: Suppl. 51, 38s. 